\title{
PharmaNews
}

Onkologie 33 | 11 | 10

\section{Finale Studien-Rekrutierungsphase}

\section{Monitor G-CSF beleuchtet Neutropenieprophylaxe}

«Bisher gibt es wenig Erkenntnisse darüber, inwieweit die Prophylaxe der febrilen Neutropenie bei Krebspatienten in Einklang mit den verfügbaren Leitlinien in der Praxis durchgeführt wird und welchen Einfluss dies auf die Behandlungsergebnisse hat», konstatierte Professor Dr. Carsten Bokemeyer im Rahmen der DGHO-Jahrestagung in Berlin. Um diese Lücke zu schließen, initiiert HEXAL die internationale, prospektive, pharmakoepidemiologische Beobachtungsstudie Monitor G-CSF zum Einsatz des Granulozyten-koloniestimulierenden Faktors (G-CSF) in der Praxis. Mindestens 1000 Patienten aus 75 Zentren werden an der Studie teilnehmen. Noch können weitere Patienten eingeschlossen werden. Bei den meisten Beobach- tungsstudien steht allein die klinische Wirkung im Fokus. Monitor G-CSF geht hier einen Schritt weiter: «Wir wollen nicht nur wissen, ob eine prophylaktische G-CSF-Behandlung wirksam ist, sondern vor allem besser definieren, wann und bei welchen Patienten», so Bokemeyer, Leiter der klinischen Prüfung. Vorrangige Ziele sind die Charakterisierung der mit dem Biosimilar Filgrastim HEXAL ${ }^{\circledR}$ behandelten Patientenpopulation, die Definition der Prophylaxeschemata mit Filgrastim HEXAL ${ }^{\circledR}$ und die Identifizierung der mehrstufigen Einflussfaktoren für Unterschiede bei hämatologischen Befunden und Ergebnissen. Daneben soll erhoben werden, inwieweit die Neutropenieprophylaxe in der Praxis mit den EORTC-Leitlinien übereinstimmt. Die Patienten werden hierzu über eine Dauer von 6 Chemotherapie-Zyklen beobachtet. Einschlusskriterien für Patienten sind unter anderem: Erwachsene beiderlei Geschlechts, Diagnose eines bestimmten Tumor- typens (Brustkrebs Stadium III oder IV, Blasenkrebs Stadium III oder IV, Lungenkrebs Stadium III oder IV, metastasierter Prostatakrebs, diffuses großzelliges B-Zell-Lymphom Stadium III oder IV), geplanter Beginn des 1. Chemotherapiezyklus, mindestens 4 geplante Zyklen und Behandlung mit Filgrastim HEXAL ${ }^{\circledR}$ nach bestem klinischen Urteil des Arztes und gemäß den Angaben in der Fachinformation.

Interessierte Ärzte und Zentren wenden sich bitte direkt an: Dr. med. Bertram Ottillinger, Tel. 08024 908-2159.

\section{Literatur}

Gascón P et al.: Crit Rev Oncol/Hematol 2010. DOI:10.1016/j.critrevonc.2010.01.014.

Weitere Informationen bei HEXAL AG / Geschäftsbereich Biosimilars über Hexal Kundenservice

Tel. +498004392527

\section{Effektive Androgendeprivation durch den GnRH-Blocker Degarelix}

GnRH-Antagonisten, auch GnRH-Blocker genannt, bieten seit circa einem Jahr eine neue Möglichkeit der Androgendeprivationstherapie (ADT). Professor Kurt Miller, Berlin, sieht die theoretischen Vorteile gegenüber den LHRHAgonisten in dem fehlenden Flare-up-Phänomen, der schnellen und tiefen Testosteronabsenkung sowie den fehlenden Microsurges. Der GnRH-Blocker Firmagon ${ }^{\circledR}$ (Degarelix) bestätigte in der Zulassungsstudie CS21 [1] die schnelle und deutliche Testosteronsuppression. «Folgeanalysen der Zulassungsstudie weisen darauf hin, dass sich die theoretischen Vorteile der Antagonisten auch in klinisch relevanten Endpunkten niederschlagen», führt Miller aus. Laut Professor Fritz H. Schröder, dem Doyen unter den Urologen, Rotterdam, unterstreichen aktuelle
Studiendaten den Stellenwert einer Hormon-Therapie mit Degarelix. In einer Subgruppenanalyse der CS21-Studie erhielten 408 Patienten 364 Tage monatlich entweder 240/80 Degarelix (initiale Dosis, Erhaltungsdosis) oder 7,5 mg Leuprorelin. Besondere Beachtung wurde den beiden Laborparametern PSA (Prostataspezifisches Antigen) und ALP (Alkalische Phosphatase) geschenkt, die monatlich bestimmt wurden. Die Studienresultate belegten ein signifikant geringeres Risiko einer PSA-Progression ( $p=0,0495$ ) unter Degarelix sowie eine schnellere PSA-Response (ITT-Population). Bei Patienten mit initial relativ hohen PSA-Werten (> $20 \mathrm{ng} / \mathrm{ml}$ ) beobachtete man eine signifikant längere Zeit bis zur PSA-Progression ( $p=0,0436$ ). Es zeigte sich zudem, dass unter der Therapie mit Firmagon ${ }^{\circledR}$ insgesamt niedrigere Spiegel der ALP-Serumkonzentration - einem Marker für Knochenmetastasen - registriert wurden. Ein Anstieg der
ALP als Hinweis auf ein Therapieversagen bei Patienten mit metastasiertem PCa und solchen mit initialen PSA-Werten $\geq 50 \mathrm{ng} / \mathrm{ml}$ trat signifikant häufiger unter der Behandlung mit dem LHRH-Agonisten Leuprorelin auf ( $p=0,0137$ ).

\section{Literatur}

1 Klotz L, et al.: BBJUI 2008;102:1531-38.

Quelle

Symposium und Pressekonferenz der Ferring Arzneimittel «Testosteron - des einen Freud', des andern Leid. Welche Rolle spielt Testosteron bei PCa und bei hypogonadalen Patienten?», 62. Kongress DGU, 23. September 2010, Düsseldorf.

Weitere Informationen bei Adlexis GmbH, Birgit Klötzer

Liebherrstr. 10, 80538 München

Tel. +49 89 2020816-0, Fax -10

\section{PharmaTicker+++ PharmaTicker+++ PharmaTicker+++ PharmaTicker+++}

Novartis Pharma GmbH. Das CHMP hat eine positive Empfehlung zur EU-weiten Zulassung von Tasigna ${ }^{\circledR}$ (Nilotinib) für die Therapie erwachsener Patienten mit neu diagnostizierter Philadelphia-Chromosom-positiver chronischer myeloischer Leukämie $(\mathrm{Ph}+\mathrm{CML})$ in der chronischen Phase abgegeben. Nilotinib bietet in der CMLErstlinientherapie als bislang einziger Tyrosinkinaseinhibitor einen signifikant besseren Schutz vor Krankheitsprogression als der aktuelle Standard Glivec $^{\circledR}$ (Imatinib).

Novartis Pharma GmbH

Dr. Irene Roth

irene.roth@novartis.com
Roche Pharma AG. Die Kombination von Erlotinib $\left(\operatorname{Tarceva}^{\circledR}\right)$ mit Gemcitabin wird in der aktuellen S3-Leitlinie zur Behandlung des metastasierten Pankreaskarzinoms empfohlen. Eine aktuelle Studie zeigt nun, dass auch Capecitabin ein möglicher Kombinationspartner für den oralen EGFRInhibitor ist. Unabhängig vom Kombinationspartner bestätigen verschiedene Studien, dass der Erlotinib-induzierte Hautausschlag ein eindeutiger prädiktiver Marker für das Therapieansprechen ist.

Roche Pharma AG

Dr. H.-U. Jelitto

www.roche.com
Archimedes Pharma. Das internationale Spezial-Pharmaunternehmen erhält von der Europäischen Kommission die Zulassung für das Produkt PecFent ${ }^{\circledR}$ (früher NasalFent). PecFent ${ }^{\circledR}$ ist ein innovatives Fentanyl-Nasenspray zur Behandlung von Tumor-Durchbruchschmerzen bei Erwachsenen, die bereits eine Basis-Schmerztherapie mit Opioiden erhalten. Die Zulassung basiert auf dem bisher größten klinischen Entwicklungsprogramm für Tumor-Durchbruchschmerzen mit 3 Phase-III-Studien.

Archimedes Pharma Germany GmbH

Dr. Armin Rath

arminrath@archimedespharma.com 